\title{
Synthesis of the Supervising Agent in MAS
}

\author{
František Čapkovič ${ }^{\star}$ \\ Institute of Informatics, Slovak Academy of Sciences \\ Dúbravská cesta 9, 84507 Bratislava, Slovak Republic \\ Frantisek.Capkovic@savba.sk \\ http://www.ui.sav.sk/home/capkovic/capkhome.htm
}

\begin{abstract}
The systematic approach to the synthesis of the agent-supervisor supervising a group of agents is presented. It is based on results of the DES (discrete event systems) control theory. The agents as well as the agent-supervisor are understood to be DES and they are modelled by Petri nets $(\mathrm{PN})$. The method based on PN place invariants is used in order to synthetise the supervisor. The illustrative example is introduced to explain the elementary steps of the proposed technique. A counterexample illustrating the limits of the approach is introduced too.
\end{abstract}

Keywords: Agent, invariants, MAS, modelling, Petri nets, supervisor.

\section{Introduction}

Agents are usually understood to be persistent (not only software, but also material, personal, etc.) entities that can perceive, reason, and act in their environment, and communicate with other agents. Hence, multiagent systems (MAS) can be apprehended as a composition of collaborative agents working in shared environment. The agents together perform a more complex functionality. Communication enables the agents in MAS to exchange information. Thus, the agents can coordinate their actions and cooperate with each other. However, an important question arises here: What communication mechanisms enhance the cooperation between communicating agents?

In this paper the synthesis of the supervising agent is presented. It utilizes some results of the DES (discrete event systems) control theory, especially those concerning the theory of place invariants 86 and the supervisor 9] and/or controller synthesis based on this theory. Mathematical and/or graphical description of the agents as well as the global MAS will be delt on the background of place/transition Petri nets $(\mathrm{P} / \mathrm{T} \mathrm{PN})$ described e.g. in [8].

Consider two simple agents $A_{1}, A_{2}$ representing, respectively, two processes $P_{1}, P_{2}$ given in Fig. 1 1 ). While the resources used by the agents are sufficient, the agents can work autonomously. However, when the resources are limited a coordinated action is necessary. In such a case a supervisor or a controller is necessary. The procedure of the mutex (mutual exclusion) frequently occurs in

* Partially supported by the Slovak Grant Agency for Science (VEGA) under grant \# 2/6102/26. 
different systems when it is necessary to solve conflicts among parallelly operating subsystems. It is very frequent also in DES like flexible manufacturing systems (e.g. a robot serves two transport belts), communication systems (two users want to use the same channel), transport systems (two trains want to enter the same segment of the railway), etc. The PN model of the simplest form of the mutex - the mutex of two processes - is given in Fig. (1)). It is not very complicated to synthesize such a supervisor in this simple case. Contrariwise, in case of more agents and more complicated conditions for co-

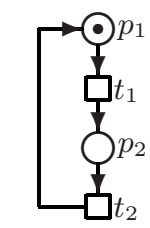

Process $P_{1}$

a)

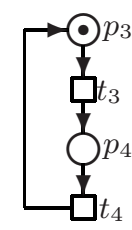

Process $P_{2}$

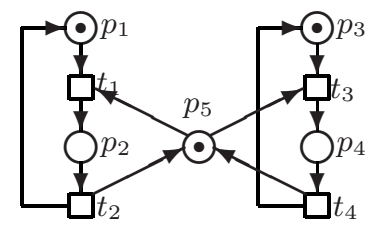

b)

Fig. 1. The PN-based model of the a) two simple processes and b) their mutex

operation among them, founding the supervisor is by far more intricated. Consider the group of 5 autonomous agents $G r_{A}=\left\{A_{1}, A_{2}, A_{3}, A_{4}, A_{5}\right\}$ given in Fig 2 Consider e.g the situation when it is necessary to ensure that only one agent from the subgroup $\operatorname{Sgr}_{1}=\left\{A_{1}, A_{4}, A_{5}\right\}$ and only one agent from the subgroup $\operatorname{Sgr}_{2}=\left\{A_{2}, A_{4}, A_{5}\right\}$ and only one agent from the subgroup $S_{g r} r_{3}=\left\{A_{3}, A_{4}, A_{5}\right\}$ can simultaneously cooperate with other agents from $G r_{A}$. In other words, the agents inside the designated subgroups must not work simultaneously. Even, the agents $A_{4}$ and $A_{5}$ can work only individually (any cooperation with other agents is excluded). However, the agents $A_{1}, A_{2}, A_{3}$ can work simultaneously. In such a situation the synthesis of the supervisor realizing such a mutex (i.e. deciding which agents can simultaneously work and when) is not so simple. Consequently, a systematic (orderly, not stray) approach has to

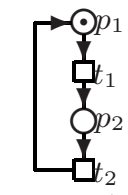

Agent $A_{1}$

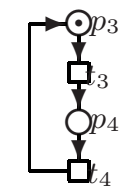

Agent $A_{2}$

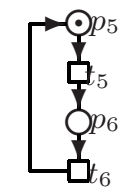

Agent $A_{3}$

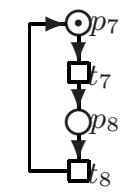

Agent $A_{4}$

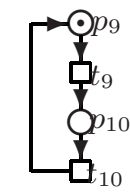

Agent $A_{5}$

Fig. 2. The PN-based model of the group of 5 simple agents

be found. There exists the DES control synthesis method based on the PN place invariants (P-invariants) in DES control theory 11/7/2/4. Its principle can be utilised for our purposes. 


\section{The Supervisor Synthesis}

Before starting the supervisor synthesis it is necessary to introduce the mathematical model of P/T PN. Namely, the ability to express the states of agents, the ability to test properties by means of the PN invariants and the reachability graph $(\mathrm{RG})$ as well as the ability to synthesize the supervisory controllers by means of the invariants and RG [3,5] utilizing linear algebra predestinate PN to be frequently used. The model has the form as follows

$$
\begin{aligned}
\mathbf{x}_{k+1} & =\mathbf{x}_{k}+\mathbf{B} \cdot \mathbf{u}_{k} \quad, \quad k=0, N \\
\mathbf{B} & =\mathbf{G}^{T}-\mathbf{F} \\
\mathbf{F} . \mathbf{u}_{k} & \leq \mathbf{x}_{k}
\end{aligned}
$$

where $k$ is the discrete step of the dynamics development; $\mathbf{x}_{k}=\left(\sigma_{p_{1}}^{k}, \ldots, \sigma_{p_{n}}^{k}\right)^{T}$ is the $n$-dimensional state vector of DEDS in the step $k ; \sigma_{p_{i}}^{k} \in\left\{0, c_{p_{i}}\right\}, i=1, \ldots, n$ express the states of the DEDS elementary subprocesses or operations - 0 (passivity) or $0<\sigma_{p_{i}} \leq c_{p_{i}}$ (activity); $c_{p_{i}}$ is the capacity of the DEDS subprocess $p_{i}$ as to its activities; $\mathbf{u}_{k}=\left(\gamma_{t_{1}}^{k}, \ldots, \gamma_{t_{m}}^{k}\right)^{T}$ is the $m$-dimensional control vector of the system in the step $k$; its components $\gamma_{t_{j}}^{k} \in\{0,1\}, j=1, \ldots, m$ represent occurring of the DEDS elementary discrete events (e.g. starting or ending the elementary subprocesses or their activities, failures, etc. - 1 (presence) or 0 (absence) of the corresponding discrete event; B, F, G are structural matrices of constant elements; $\mathbf{F}=\left\{f_{i j}\right\}_{n \times m}, f_{i j} \in\left\{0, M_{f_{i j}}\right\}, i=1, \ldots, n, j=1, \ldots, m$ expresses the causal relations among the states of the DEDS (in the role of causes) and the discrete events occuring during the DEDS operation (in the role of consequences) - 0 (nonexistence), $M_{f_{i j}}>0$ (existence and multiplicity) of the corresponding causal relations; $\mathbf{G}=\left\{g_{i j}\right\}_{m \times n}, g_{i j} \in\left\{0, M_{g_{i j}}\right\}, i=1, \ldots, m, j=1, \ldots, n$ expresses very analogically the causal relations among the discrete events (causes) and the DEDS states (consequences); because $\mathbf{F}$ and $\mathbf{G}$ are the arcs incidence matrices the matrix $\mathbf{B}$ is given by means of them according to (2); $(.)^{T}$ symbolizes the matrix or vector transposition.

The main idea of the approach to the supervisor synthesis consists in imbedding of additional PN places (slacks) and finding the structural interconnections between them and the original PN places. Consequently, a new PN subnet representing the supervisor and its interface with the original system are found and added to the PN model. Thus, the desired behaviour of agents is forced. The definition of the $\mathrm{P}$-invariant $\mathbf{v}$ of $\mathrm{PN}$ in general is the following

$$
\mathbf{B}^{T} \cdot \mathbf{v}=\emptyset
$$

with $\mathbf{v}$ being $n$-dimensional vector and $\emptyset$ is $m$-dimensional zero vector. However, usually there are several invariants in PN models. Hence, the set of the $\mathrm{P}$-invariants of $\mathrm{PN}$ is created by the columns of the $n \times n_{x}$-dimensional $\left(n_{x}\right.$ expresses the number of invariants) matrix $\mathbf{V}$ being the solution of the equation

$$
\mathbf{V}^{T} \cdot \mathbf{B}=\emptyset
$$


In $\mathrm{PN}$ with the slacks mentioned above we have to use the following structure with augmented matrices. Consequently, the equation (5) has to be writen in the form

$$
\left[\mathbf{L}, \mathbf{I}_{s}\right] \cdot\left[\begin{array}{l}
\mathbf{B} \\
\mathbf{B}_{s}
\end{array}\right]=\boldsymbol{\emptyset}
$$

where $\mathbf{I}_{s}$ is $\left(n_{x} \times n_{x}\right)$-dimensional identity matrix and $\mathbf{L}$ describes conditions (as to marking of some places in the PN models of agents) of the desired MAS behaviour. While $\mathbf{B}$ is the known structural matrix of the agents (without the supervisor), $\mathbf{B}_{s}$ is the supervisor structure which is searched by the synthesis process. Thus,

$$
\mathbf{L} . \mathbf{B}+\mathbf{B}_{s}=\emptyset ; \quad \mathbf{B}_{s}=-\mathbf{L} . \mathbf{B} ; \quad \mathbf{B}_{s}=\mathbf{G}_{s}^{T}-\mathbf{F}_{s}
$$

where the actual structure of the matrix $\mathbf{L}$ has to be respected. The augmented state vector and the augmented structural matrices (of the original system together with the supervisor) are the following

$$
\mathbf{x}_{a}=\left[\begin{array}{l}
\mathbf{x} \\
\mathbf{x}_{s}
\end{array}\right] ; \quad \mathbf{F}_{a}=\left(\begin{array}{l}
\mathbf{F} \\
\mathbf{F}_{s}
\end{array}\right) ; \quad \mathbf{G}_{a}^{T}=\left(\begin{array}{l}
\mathbf{G}^{T} \\
\mathbf{G}_{s}^{T}
\end{array}\right)
$$

where the submatrices $\mathbf{F}_{s}$ and $\mathbf{G}_{s}^{T}$ correspond to the interconnections of the incorporated slacks with the actual (original) PN structure.

\subsection{The Illustrative Example}

It can be seen that in the above mentioned case of the five agents the form of $\mathbf{L}$ follows from the imposed conditions prescribing the limited cooperation of agents expressed mathematically as

$$
\begin{aligned}
\sigma_{p_{2}}+\sigma_{p_{8}}+\sigma_{p_{10}} & \leq 1 \\
\sigma_{p_{4}}+\sigma_{p_{8}}+\sigma_{p_{10}} & \leq 1 \\
\sigma_{p_{6}}+\sigma_{p_{8}}+\sigma_{p_{10}} & \leq 1
\end{aligned}
$$

The form of the augmented matrix $\left[\mathbf{L}, \mathbf{I}_{s}\right]$ follows from the modified conditions (introducing the slacks) which are given mathematical terms as

$$
\begin{array}{rrr}
\sigma_{p_{2}}+\sigma_{p_{8}}+\sigma_{p_{10}}+\sigma_{p_{11}} & =1 \\
\sigma_{p_{4}}+\sigma_{p_{8}}+\sigma_{p_{10}}+\sigma_{p_{12}} & =1 \\
\sigma_{p_{6}}+\sigma_{p_{8}}+\sigma_{p_{10}} & +\sigma_{p_{13}} & =1
\end{array}
$$

where $p_{11}, p_{12}, p_{13}$ are the additional places - i.e. the slacks $s_{1}, s_{2}, s_{3}$ (in general, the number $i$ of such slacks $s_{i}$ can also be different from $i=3$, of course) - that ensure the desired eliminations of the agents cooperation activities. The number of the slacks depends on the number of conditions and on their form. As it is clear from the equations (10)-(12), only one place from the set of four places 
$p_{2}, p_{8}, p_{10}, p_{11}$ can be active while the rest ones have to be passive. Analogically, the same is valid for the sets $p_{4}, p_{8}, p_{10}, p_{12}$ and $p_{6}, p_{8}, p_{10}, p_{13}$.

$$
\begin{aligned}
& \mathbf{F}=\left(\begin{array}{lllll}
\mathbf{F}_{A_{1}} & \mathbf{0} & \mathbf{0} & \mathbf{0} & \mathbf{0} \\
\mathbf{0} & \mathbf{F}_{A_{2}} & \mathbf{0} & \mathbf{0} & \mathbf{0} \\
\mathbf{0} & \mathbf{0} & \mathbf{F}_{A_{3}} & \mathbf{0} & \mathbf{0} \\
\mathbf{0} & \mathbf{0} & \mathbf{0} & \mathbf{F}_{A_{4}} & \mathbf{0} \\
\mathbf{0} & \mathbf{0} & \mathbf{0} & \mathbf{0} & \mathbf{F}_{A_{5}}
\end{array}\right) ; \mathbf{G}=\left(\begin{array}{lllll}
\mathbf{G}_{A_{1}} & \mathbf{0} & \mathbf{0} & \mathbf{0} & \mathbf{0} \\
\mathbf{0} & \mathbf{G}_{A_{2}} & \mathbf{0} & \mathbf{0} & \mathbf{0} \\
\mathbf{0} & \mathbf{0} & \mathbf{G}_{A_{3}} & \mathbf{0} & \mathbf{0} \\
\mathbf{0} & \mathbf{0} & \mathbf{0} & \mathbf{G}_{A_{4}} & \mathbf{0} \\
\mathbf{0} & \mathbf{0} & \mathbf{0} & \mathbf{0} & \mathbf{G}_{A_{5}}
\end{array}\right) \\
& \mathbf{F}_{A_{i}}=\left(\begin{array}{ll}
1 & 0 \\
0 & 1
\end{array}\right) ; \mathbf{G}_{A_{i}}=\left(\begin{array}{ll}
0 & 1 \\
1 & 0
\end{array}\right) ; \mathbf{B}_{A_{i}}=\left(\begin{array}{rr}
-1 & 1 \\
1 & -1
\end{array}\right) ; i=1, \ldots, 5
\end{aligned}
$$

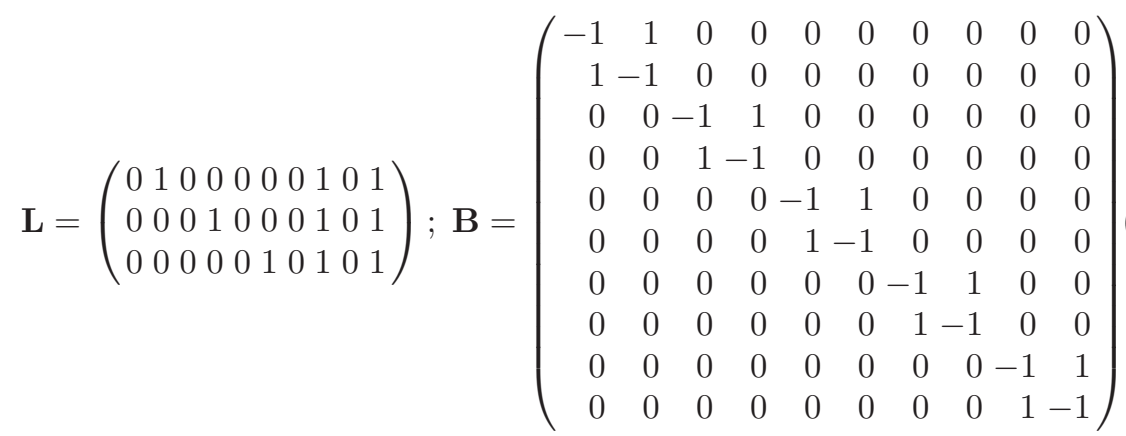

$$
\begin{aligned}
& \mathbf{B}_{s}=-\mathbf{L} \cdot \mathbf{B}=\left(\begin{array}{rrrrrrrr}
-1 & 1 & 0 & 0 & 0 & 0-1 & 1-1 & 1 \\
0 & 0 & -1 & 1 & 0 & 0-1 & 1-1 & 1 \\
0 & 0 & 0 & 0 & -1 & 1-1 & 1-1 & 1
\end{array}\right)
\end{aligned}
$$

Hence, by wirtue of (6), we have

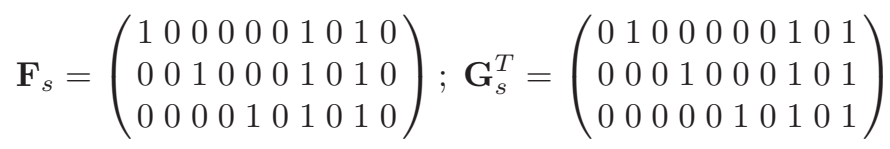

Thus, we obtain the structure of MAS given in Fig. 3 where the supervisor is represented by the structure consisting of the places $p_{11}, p_{12}, p_{13}$ together with the interconnections joining them with the elementary agents. Such MAS exactly fulfils the prescribed conditions. The reachability graph (RG) 8 of the system is given in Fig. 4 Its nodes are the states reachable from the initial state $\mathbf{x}_{0}$ and its edges are weighted by corresponding PN transitions enabling the state-to-state transit. The control synthesis based on RG is described in the author's works 35. Consequently, this problem is not discussed here. In the light of the above introduced facts we can illustrate also the systematic synthesis of the simplest mutex presented in Fig. 1. Here we have

$$
\begin{array}{ll}
\sigma_{p_{2}}+\sigma_{p_{4}} & \leq 1 \\
\sigma_{p_{2}}+\sigma_{p_{4}}+\sigma_{p_{5}} & =1
\end{array}
$$




$$
\begin{gathered}
\mathbf{L}=(0,1,0,1) ; \quad \mathbf{B}=\left(\begin{array}{rrrr}
-1 & 1 & 0 & 0 \\
1 & -1 & 0 & 0 \\
0 & 0 & -1 & 1 \\
0 & 0 & 1 & -1
\end{array}\right) ; \quad \mathbf{L} \cdot \mathbf{B}=(1,-1,1,-1) \\
\mathbf{B}_{s}=-\mathbf{L} \cdot \mathbf{B}=(-1,1,-1,1) ; \quad \mathbf{F}_{s}=(1,0,1,0) ; \quad \mathbf{G}_{s}^{T}=(0,1,0,1)
\end{gathered}
$$

Of course, the result is the same like that given in the Fig. 1, b) - compare (21) with Fig. 1]).

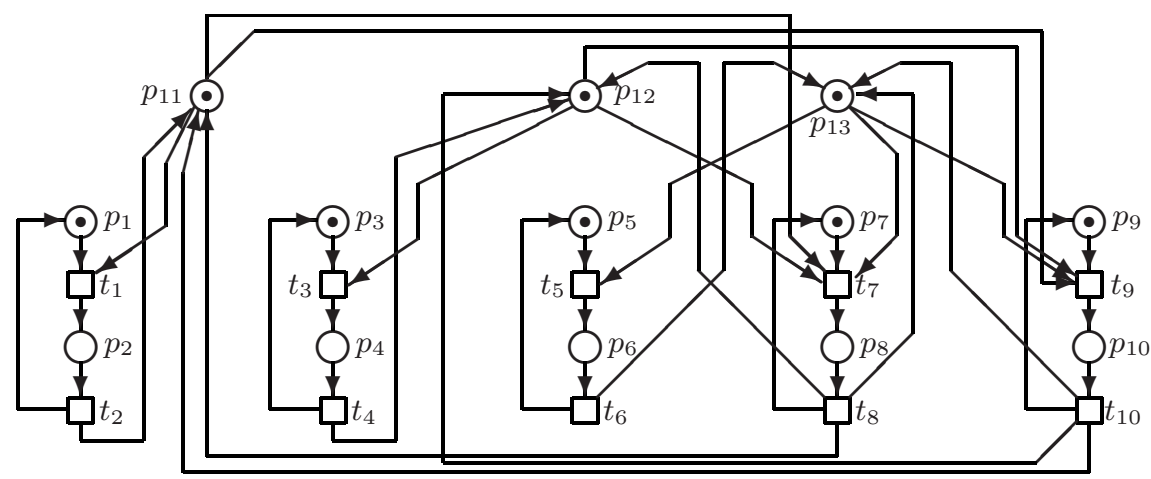

Fig. 3. The PN-based model of the controlled cooperation of the agents
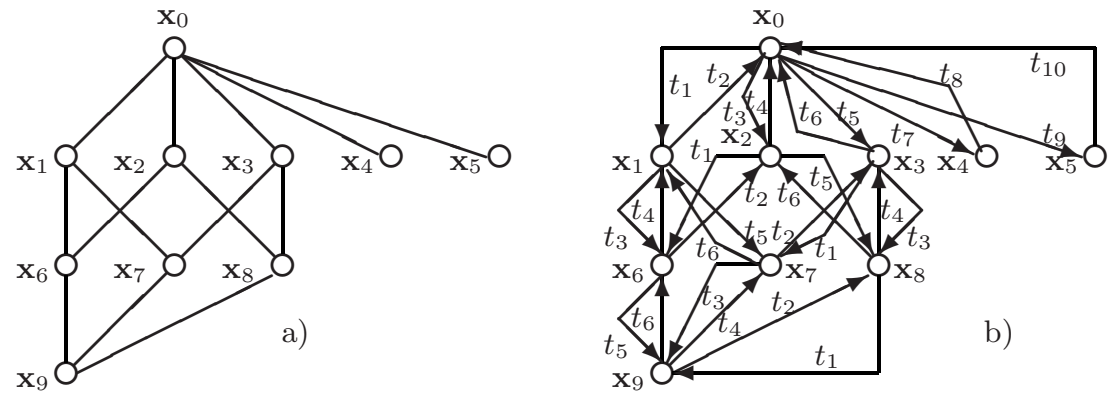

Fig. 4. The RG of the five cooperating agents a) in the form where the edges are oriented bidirecitonally, but weights of the elementary edges are not introduced because they are different depending on the orientation; b) in the form where both the orientation of edges and their weights are introduced implicitly

\section{Applicability of the Approach}

The method described above is suitable for the so called pure PN. P/T PN is pure when there is no 'loop' between a place and a transition - i.e. it is the net without the loops of the form given in Fig. 5 a). Namely, in such a case 
the matrices $\mathbf{F}, \mathbf{G}^{T}$ have nonzero element equal to 1 in the same place - in general, when there exists the 'loop' between $p_{i}$ and $t_{j}$ the element $f(i, j)$ of the matrix $\mathbf{F}$ is equal to the element $g T(i, j)$ of the matrix $\mathbf{G}^{T}$. The effect is, that the difference $\mathbf{G}^{T}-\mathbf{F}=\mathbf{B}$ gives us a deformed information about the system structure, because the elements $f(i, j), g T(i, j)$ eleminate each other (namely, $g T(i, j)-f(i, j)=0)$. Fortunately, in PN theory a simple solution of such a problem is possible. It is sufficient to replace the transition in question by the fictive structure introduced in Fig. 5 b). The auxiliary place $p^{\prime}$ together with its input and output transitions $t^{\prime}$ and $t^{\prime \prime}$ replace the original transition $t$. It means that the impureness in the form of a 'loop' can be replaced (without changing any dynamic property of the net) very simply by the replacement of the transition in question by two fictive transitions and a fictive place set between them. Such a replacement is invariant. After removing the 'loop' problems the original method of the supervisor synthesis can be utilized also for the systems modelled by the 'impure' P/T PN.

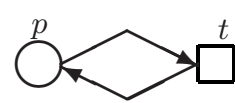

a)

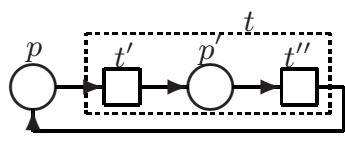

b)

Fig. 5. The replacement of the loop. The original loop is on the left. The structure replacing the loop is given on the right.

\subsection{Dealing with Problems of Mutual Interactions Among Agents}

Many times, another form of demands on the cooperation among agents occurs. Especially, the situation - see e.g. [1] - when there exist mutual interactions among the agents to be supervised. In such a case a particular marking (i.e. the state of a concrete place) of one agent affects (e.g. stops) firing a transition in another agent. The situation is illustrated in Fig. 6a), where the transition ${ }^{A_{1}} t$ of the agent $A_{1}$ is prevented from firing under marking the place ${ }^{A_{2}} p$ of the agent $A_{2}$. As we can see in that picture, a figment that the place $p^{\prime}$ represents the supervising agent $A_{3}$ (or, better said, its part) can be accepted and consecutively, the above described approach to the supervisor synthesis can be utilized. Namely, the transition ${ }^{A_{1}} t$ can be substituted by means of the auxilary fictive place $p^{\prime \prime}$ together with its fictive input and output transitions $t^{\prime}$, and $t^{\prime \prime}$ analogically to that displayed in Fig. 5b). Hence, the new situation - i.e. new structure is displayed in Fig 6b). It can be seen that it is the classical mutex. Such a substitution can be utilized in synthesis of the supervisor in case of agents with more complicated structure. For example, the well known kind of the readerwriter cooperation named as the Owicki/Lamports mutex (defined e.g. in [10]) can be automatically synthetised using this substitution.

However, it is necessary to say, that there exist cases where the synthesis of the supervisor based on invariants is not so simple (i.e. fully automatized) or even, it is impossible. Here, the experience and invention of the creator of the 


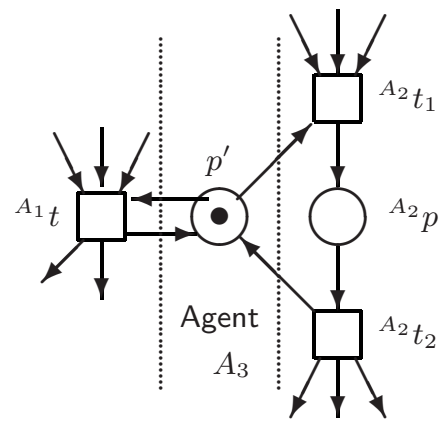

Agent $A_{1}$
Agent $A_{2}$

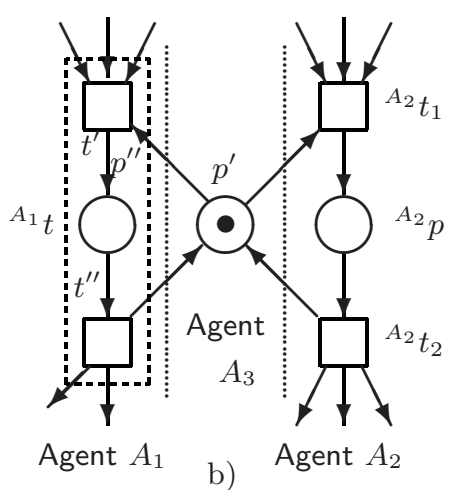

b)

Fig. 6. The prevention of the transition ${ }^{A_{1}} t$ (the part of the Agent $A_{1}$ ) from firing under the marking ${ }^{A_{2}} p$ (the part of the Agent $A_{2}$ ) by means of $p^{\prime}$ - the part of the supervisor, i.e. the Agent $A_{3}$. a) The original schema; b) The substitutive schema.

agents supervisor has to be used. In the next illustrative example such a system is presented and described.

\section{$3.2 \quad$ The Counter-Example}

In order to illustrate such a counter-example let us consider the so called Peterson's mutex algorithm [10] given in Fig. 7 This system consists of two agents $A_{1}$ (writer) and $A_{2}$ (reader). The PN places describe the following activities: $p_{1}$ - pending 1 of $A_{1} ; p_{2}$ - pending 2 of $A_{1} ; p_{3}$ - critical $A_{1} ; p_{4}$ - finished $A_{1} ; p_{5}$ pending 0 of $A_{1} ; p_{6}$ - quiet $A_{1} ; p_{7}$ - pending 1 of $A_{2} ; p_{8}$ - pending 2 of $A_{2} ; p_{9}$ critical $A_{2} ; p_{10}$ - finished $A_{2} ; p_{11}$ - pending 0 of $A_{2} ; p_{12}$ - quiet $A_{2} ; p_{13}$ - at $A_{1}$ (left); $p_{14}$ - at $A_{2}$ (right).

The state $p_{4}$ signals to $A_{2}$ that $A_{1}$ is presently not striving to become critical. This allows $A_{2}$ to "easily" access its critical region, by the action represented by the transition $t_{12}$. Likewise, the state $p_{10}$ allows $A_{1}$ to access its critical state, by the action represented by the transition $t_{5}$. The shared token alternates between the states $p_{13}$ and $p_{14}$ The step from $p_{1}$ to $p_{2}$ results in the token in $p_{13}$ : by action expressed by the transition $t_{2}$ in case $A_{1}$ obtains the token from $p_{13}$, or by action expressed by the transition $t_{3}$ in case at $A_{1}$ held the token anyway. The step from $p_{7}$ to $p_{8}$ likewise results in the token in $p_{14}$. Hence, the token is always at the site that executed the step from 'pending 1' to 'pending 2' most recently.

After leaving $p_{6}$ along the quiescent action represented by the transition $t_{7}$, the $A_{1}$ takes three steps to reach its critical state $p_{3}$. In the first step, the fair action represented by the transition $t_{6}$ brings $A_{1}$ from $p_{5}$ to $p_{1}$ and removes the state $p_{4}$. Fairness of the transition $t_{6}$ is local, because the transition $t_{6}$ is local to $A_{1}$, with $p_{4}$ the only forward branching the place in the transition $t_{6}$, which is connected to the $A_{2}$ by the loop $\left(p_{4}, t_{12}\right)$. The second step, from $p_{1}$ to $p_{2}$, results in the shared token in the place $p_{13}$, as described above. The third step brings $A_{1}$ to $p_{3}$, with action expressed by the transition $t_{5}$ in case $A_{2}$ signals with $p_{10}$, 


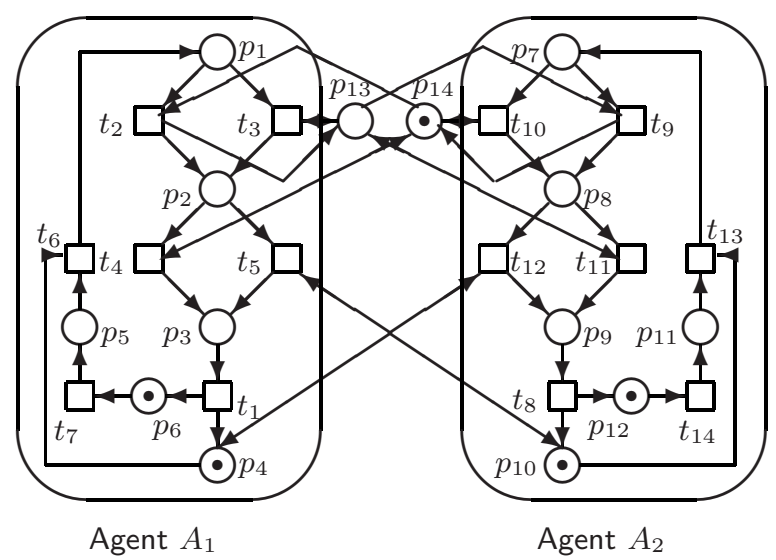

Fig. 7. The PN-based model of the two agents communication - the writer (agent A1) and the reader (agent A2) - in the form of the Peterson's mutex

that it is presently not interested in going critical, or with action represented by the transition $t_{4}$ in case $A_{2}$ more recently executed the step from $p_{7}$ to $p_{8}$.

The algorithm's overall structure guarantees that one of $p_{10}$ or $p_{14}$ will eventually carry a token that remains there until eventually either the event represented by $t_{5}$ or $t_{4}$ occurs. The two agents are structurally symmetrical, but the initial state favors $A_{2}$ (if the place $p_{14}$ contains the token) or $A_{1}$ (if the place $p_{13}$ contains the token).

In this example the invariants-based approach to the supervisor synthesis fails. Namely, any satisfying matrix $\mathbf{L}$ cannot be found. Searching for reasons it was detected that the problem consists in the specific structure of the P-invariants of the global system presented in Fig. 7. As we can see from the following matrix of the global system $\mathrm{P}$-invariants the invariants of the agents $A_{1}, A_{2}$ are mutually disjoint

$$
\mathbf{V}^{T}=\left(\begin{array}{llllllllllllll}
0 & 0 & 0 & 0 & 0 & 0 & 1 & 1 & 1 & 0 & 1 & 1 & 0 & 0 \\
0 & 0 & 0 & 0 & 0 & 0 & 1 & 1 & 1 & 1 & 0 & 0 & 0 & 0 \\
0 & 0 & 0 & 0 & 0 & 0 & 0 & 0 & 0 & 0 & 0 & 0 & 1 & 1 \\
1 & 1 & 1 & 0 & 1 & 1 & 0 & 0 & 0 & 0 & 0 & 0 & 0 & 0 \\
1 & 1 & 1 & 1 & 0 & 0 & 0 & 0 & 0 & 0 & 0 & 0 & 0 & 0
\end{array}\right)
$$

\section{Conclusion}

The method of the DES control theory based on PN place invariants was utilized for the synthesis of the agent (with prescribed properties) supervising a group of agents in MAS. The illustrative example documenting the soundness of the method was introduced. It was shown that the method is simply applicable for autonomous agents modelled by pure PN. Additionally, it was described how the applicability can be extended for the agents modelled by impure PN. Namely, 
a simple substitution of the transition in the loops of the PN model is sufficient to remove the impureness. As a result of the research it can be said that the method is very useful for the synthesis of the agents supervising a group of automous agents. In case of a group of cooperating agents some problems can occur. Therefore, the counter-example was presented. It was shown that e.g. for the reader-writer in the form of the so called Peterson's mutex the supervising agent cannot be synthetised by the proposed method. Hence, there exists a challenge for the future investigation on this way, especially, to define general conditions for the validity of the approach.

\section{References}

1. Bordbar, B., Giacomini, L., Holding, D.J.: UML and Petri Nets for Design and Analysis of Distributed Systems. In: IEEE International Conference on Control Applications (CCA 2000) and IEEE International Symposium on Computer-Aided Control System Design (CACSD 2000), pp. 610-615. IEEE Press, Piscataway (2000)

2. Buy, U., Darabi, H.: Sidestepping Verification Complexity with Supervisory Control. In: Workshop on Software Engineering for Embedded Systems: From Requirements to Implementation, 8 pages (2003), http://www.cs.uic.edu/ shatz/SEES/Schedule.htm

3. Capkovič, F.: An Application of the DEDS Control Synthesis Method. Journal of Universal Computer Science 11, 303-326 (2005)

4. Čapkovič, F.: DES Modelling and Control vs. Problem Solving Methods. Int. J. Intelligent Information and Database Systems 1, 53-78 (2007)

5. Capkovič, F.: Modelling, Analysing and Control of Interactions among Agents in MAS. Computing and Informatics 26, 507-541 (2007)

6. Martinez, J., Silva, M.: A simple and fast algorithm to obtain all invariants of generalized Petri net. In: Application and Theory of Petri Nets, vol. 52, pp. 301311. Springer, New York (1982)

7. Moody, J.O., Antsaklis, P.J.: Supervisory Control of Discrete Event Systems Using Petri Nets. Kluwer Academic Publishers, Boston (1998)

8. Murata, T.: Petri Nets: Properties, Analysis and Applications. Proceedings IEEE 77, 541-580 (1989)

9. Ramadge, P.J., Wonham, W.M.: Supervisory Control of a Class of Discrete Event Processes. SIAM Journal on Control and Optimization 25, 206-230 (1987)

10. Reisig, W.: Elements of Distributed Algorithms. Modeling and Analysis with Petri Nets. Springer, Berlin (1998)

11. Yamalidou, K., Moody, J.O., Antsaklis, P.J., Lemmon, M.D.: Feedback Control of Petri Nets Based on Place Invariants. Automatica 32, 15-28 (1996) 